\title{
In vitro chemosensitivity in ovarian carcinoma: Comparison of three leading assays
}

\author{
Burak Tatar', Gökhan Boyraz², İlker Selçuk ${ }^{3}$, Alper K. Doğan ${ }^{4}$, Alp Usubütün ${ }^{5}$, Zafer Selçuk Tuncer ${ }^{2}$ \\ ${ }^{1}$ Department of Gynecologic Oncology, Süleyman Demirel University School of Medicine, Isparta, Turkey \\ ${ }^{2}$ Department of Gynecologic Oncology, Hacettepe University School of Medicine, Ankara, Turkey \\ ${ }^{3}$ Department of Gynecologic Oncology, Zekai Tahir Burak Maternity and Teaching Hospital, Ankara, Turkey \\ ${ }^{4}$ Onkosel Biotechnology, Hacettepe University Technopolis, Ankara, Turkey \\ ${ }^{5}$ Department of Pathology, Hacettepe University School of Medicine, Ankara, Turkey
}

\section{Abstract}

Objective: An alternative approach to the current therapy of ovarian carcinoma is the individualization of treatment by determining the sensitivity of tumoral tissue to chemotherapeutic agents before the initiation of chemotherapy. The objectives of the study are to determine the efficacy of in vitro chemosensitivity assays in ovarian carcinoma and to measure the correlation of three leading assays.

Material and Methods: Fresh tumoral tissue samples of 26 newly diagnosed primary ovarian cancer patients were studied with 3-(4,5-dimethylthiazol-2-yl)-2,5-diphenyltetrazolyum bromide (MTT) assay, adenosine triphosphate-tumor chemosensitivity assay (ATP-TCA) and differential staining cytotoxicity (DISC) assays. Chemosensitivity of tumors were studied for paclitaxel, carboplatin, docetaxel, topotecan, gemcitabine, and doxorubicin with each of the three assays. Subgroup analysis was performed for stage, grade, and histologic type.

Results: The in vitro chemosensitivity results of MTT, ATP, and DISC assays were found to be similar. The subgroups in which in vitro assays would be more useful were encountered for patients with advanced stage and serous histology ovarian carcinoma.

Conclusions: In vitro chemosensitivity can be determined in ovarian carcinoma with ATP, MTT, or DISC assays before the initiation of chemotherapy. These three assays correlate well with each other and are particularly useful for serous and advanced cancers. Large prospective studies comparing standard versus assay-directed therapy with an endpoint of overall survival are required before routine clinical utilization of these assays. (J Turk Ger Gynecol Assoc 2016; 17: 35-40)

Keywords: Ovarian cancer, in vitro chemosensitivity, ATP, MTT, DISC

Received: 20 January, 2016

Accepted: 22 January, 2016

\section{Introduction}

Ovarian cancer is still one of the most difficult cancers to combat, and the mortality rate is high (1). Epithelial histologic type constitutes $90 \%$ of all ovarian cancer cases (2). Because there is no effective screening test for early detection, threefourths of cases are still in the advanced stage at presentation. The standard therapy basically consists of surgical resection of the tumor mass followed by chemotherapy that targets residual disease. Except for early-stage and well-differentiated tumors, all patients receive a first-line adjuvant chemotherapy regimen including carboplatin and paclitaxel (3, 4). Surgery followed by combination chemotherapy, which is based on evidence from previous clinical trials, reveals a response rate of approximately $70-80 \%$ (5). However, most patients will have a relapse in the follow-up, and only-one third will survive after 5 years (6). As the disease recurs, there is no standard for therapy and no single treatment regimen has significant impact on overall survival (OS) (7).

All the patients with ovarian carcinoma may not have equal response to the standard chemotherapeutic regimen, although they have the same histologic type of tumor. The main factor causing this difference is thought to be the heterogeneity of the tumor tissue. Thus, histologic assessment yet does not provide data for the chemotherapeutic response. Furthermore, ploidy analyses, assessment of the proliferation rate of tumor cells, or determination of oncogens does not have a reliable correlation with the chemotherapeutic response. The sensitivity of a tumor to a chemotherapy regimen may only be determined after the completion of several cycles of cytotoxic drugs. An alternative approach to current therapy is the individualization of treatment by determination of the sensitivity of tumor tissue to chemotherapeutic agents before the initiation of chemotherapy (8). To date, chemosensitivity comes around by the need for selection of sensible 
agents to the tumor and for the application of most efficient chemotherapeutic drugs.

The individualized chemotherapy methods are the area of research since the pioneering studies of Black and Speer in 1950s (9). The chemosensitivity tests allow detecting the cytotoxic, cytostatic, and apoptotic effects of the chemotherapeutic agent outside the organism. Such an approach is particularly thought to prevent the harmful toxic effects of these agents. Many molecular and cellular assays have been developed to date for the in vitro detection of chemosensitivity. The molecular methods detect the chemosensitivity at the protein or gene level $(10,11)$. Although a single gene may be sufficient for the evaluation, a cytotoxic drug sometimes generates an excessive cellular response $(12,13)$. Cellular methods provide the efficacy results for multiple drugs simultaneously. Several tests based on cellular methods, including 3-(4,5-dimethylthiazol2-yl)-2,5-diphenyltetrazolyum bromide (MTT) chemosensitivity assay, adenosine triphosphate-tumor chemosensitivity assay (ATP-TCA), and differential staining cytotoxicity (DISC) assays, have been described in the literature.

Despite the logic that any method that predicts the chemosensitivity of a tumor for an individual patient would be helpful for better regimens, no chemosensitivity assay has achieved widespread clinical use to date. In a study from USA with 262 patients, all the patients were treated empirically, but a chemosensitivity assay was also performed concomitantly for all. In addition, in the subgroup of patients treated with assay-sensitive agents, progression-free survival (PFS) and OS are found to be improved, and further analysis has confirmed these results $(14,15)$. Recently, an observational study has also evaluated chemosensitivity profiles of type 1 and type 2 epithelial ovarian cancer (EOC) (16). There is a dearth of large randomized prospective trials for ovarian cancer evaluating the survival of patients treated by empirically decided therapy versus selected chemotherapeutic regimen based on the in vitro chemosensitivity assay results (assay-directed therapy).

Furthermore, no study has compared the in vitro chemosensitivity assays MTT, ATP-TCA, and DISC in the same fresh ovarian tumor samples. Thus, we aimed to determine the clinical consistency of three abovementioned leading in vitro chemosensitivity assays using a series of EOC tumors and discuss the feasibility of individualized chemotherapeutic treatment in the future.

\section{Material and Methods}

In total, 26 patients with EOC diagnosed from January 2011 to April 2012 at the Gynecologic Oncology Unit, Department of Obstetrics and Gynecology, Hacettepe University School of Medicine, Ankara, Turkey are included in this study. All patients were operated for pelvic mass and underwent to cytoreductive surgery after the frozen section confirmed epithelial ovarian carcinoma.

After frozen section evaluation of the tumor, approximately 2 $\mathrm{cm}^{3}$ of fresh tumor tissue was obtained for in vitro chemosensitivity testing. The tumor sample was transported in a plastic bottle containing liquid composed of carbon dioxide-free medium, horse serum, calf serum, penicillin-streptomycin- glutamin combination, gentamycin, and amfotericin $\mathrm{B}$. The in vitro chemosensitivity assays were performed by the co-author at Onkosel Biotechnology Company, Hacettepe Technopolis, Ankara, Turkey.

The chemosensitivity results were obtained for each patient with regard to three different assays (MTT, ATP, and DISC assays) and six different drugs (paclitaxel, carboplatin, docetaxel, topotecan, gemcitabine, and doxorubicin). Results were categorized according to the percentage of cell death after the administration of chemotherapeutic agent to the cell cultures. For the DISC assay, $\mathrm{LC}_{90}$, which represents the dose which causes $90 \%$ loss of viability in cell population, constitutes the control. For ATP and MTT tests, two controls, including the 100\% cell death after high-dose fluorouracil application group and no drug added, i.e., the $0 \%$ cell death group, were used. The remaining viable cell percentage in comparison with that in the control group without any drugs added ( $0 \%$ cell death) for each specimen was categorized as sensitive, moderately sensitive, resistant, and extremely resistant (<30\%, 30\%-49\%, 50\%-90\%, and $>90 \%$ remaining viable cells in comparison with those in the control group, respectively).

\section{ATP assay}

For the ATP assay, chemosensitivity was evaluated with the ATP-TCA kit (TCA-100; Innovative Diagnostik Systeme (DCS), Hamburg, Germany). Tumor cells acquired from tumoral tissue disintegrated first mechanically and then by enzymatic dissociation (Collagenase Worthington Type CLS III, Biochrom; Berlin, Germany). A single cell suspension was then acquired, and dissociated cells were counted to have at least 7500 viable cells. After seeding in a 96-well microplate, cells were incubated for 5 days at $37^{\circ} \mathrm{C}$ and $5 \%$ carbon dioxide and then treated with different drugs to be tested. In each plate, there were two types of control: a control without any drug added and a control of maximum inhibitor, which kills all the cells. After the incubation period, lysis of the surviving cells was performed by adding tumor cell extraction reagent (DCS Innovative Diagnostik Systeme). A sample of the suspension from each well was added to corresponding wells in another 96-well microplate. A luciferin-luciferase reagent was added, and the illumination level corresponding to ATP level was measured using a luminometer (Berthold; Hamburg, Germany) and analyzed. Luminescence is correlated with ATP levels, and this reflects the inhibition in comparison with that of untreated controls included in each plate and reported as "percent inhibition."

\section{MTT assay}

For the MTT assay, tumor samples were cut into small pieces of $5 \times 5 \mathrm{~mm}$. The tumor in the transport medium was further lysed mechanically and was put over $5 \mathrm{~mL}$ of solution (Ficoll 400, Telebrix 35, and distilled water) and centrifuged for $30 \mathrm{~min}$ at $2200 \mathrm{rpm}$. Tumor cells were collected, washed, and centrifuged again for $5 \mathrm{~min}$ at $1600 \mathrm{rpm}$. If there were red blood cells (RBCs) in pellets, RBCs were lysed by adding $5 \mathrm{~mL}$ of aqua pro. Then, washing medium was added and centrifuged for $5 \mathrm{~min}$ at $1200 \mathrm{rpm}$. Cells were resuspended at concentrations of $1 \times 106$ viable cells $/ \mathrm{mL}$ in cultivation medium and seeded in 
96-well microplates, with approximately $80 \mu \mathrm{L}$ of cell suspension per well. Following this, tumor cells were exposed to chemotherapeutics for $72 \mathrm{~h}$. Two columns were controls. After the cultivation occurred, $10 \mu \mathrm{L}$ MTT $(5 \mathrm{mg} / \mathrm{mL}$ ) was added to each well. Formazan crystals have emanated, and $100 \mu \mathrm{L}$ of $10 \%$ sodium dodecyl sulfate (SDS) solution was added. Next, the plates were assessed for absorbance at $560 \mathrm{~nm}$ using a spectrophotometer (Tecan Spectra Fluor-Plus; Salzburg, Austria).

\section{DISC assay}

For the DISC assay, a cell culture was prepared from fresh tumor tissue by enzymatic digestion. Tumor cell clusters were incubated for 3 days in 96-well microplates. Differential staining was performed with fast green dye. Following centrifugation, normal and neoplastic cells were fixed on slides. The control group without administration of any chemotherapeutic agent and complete cell death group with administration of overdose fluorouracil were compared with the group of cells exposed to a specific chemotherapeutic agent. Viable cells were detected with red or pink color, and dead cells were blue or purple (Figure 1).

\section{Statistical analysis}

Comparison of MTT, ATP, and DISC assays for chemosensitivity was performed using matched $\mathrm{t}$ test and Pearson correlation analysis to detect the power and tendency of the relation among them. For chemosensitivity analysis of agents for each test, variance analyses for recurrent measurements were used. The difference in chemosensitivity between drugs was investigated by variance analyses of recurrent measurements, and Bonferroni related t test was used. The relationship between categorical values, including chemosensitivity discrimination, in age groups was analyzed by Pearson chi-square test and Fisher's Chi-square test. The power (p) was also reported for the recurrent measurements of variance analyses. Statistical analysis was conducted by SPSS 16.0 (SPSS Inc.; Chicago, IL, USA), and $\mathrm{p}<0.05$ was determined to be significant.

This study was approved by the Hacettepe University Ethics Committee. A written informed consent was obtained from all the patients before the operation for enrollment to the study. The procedures followed were in accordance with the ethical standards of the responsible committee on human experimentation (institutional and national) and with the Helsinki Declaration of 1975, as revised in 2000 and 2008.

\section{Results}

The mean age of the patients were 56.0 years (range $41-72$ years). Of the patients, 19 (73.0\%) had serous, four had endometrioid, two had mucinous, and one had undifferentiated type of tumor. Fourteen patients had grade $3(53.8 \%)$, six had grade 2 , and six had grade 1 tumor. Sixteen patients (61.5\%) had stage III disease, four had stage II disease, and six had stage Ic disease.

The in vitro chemosensitivity of tumor cells to chemotherapeutic agents, including paclitaxel, carboplatin, docetaxel, topotecan, gemcitabine, and doxorubicin was measured by MTT, ATP, and DISC assays (Table 1).
The spectrophotometric quantitative value of ATP and MTT tests correlated with that of the DISC test for each chemotherapeutic agent. The correlation of results of MTT and DISC tests for paclitaxel $(\mathrm{r}=0.95, \mathrm{p}<0.01)$, carboplatin $(\mathrm{r}=0.94, \mathrm{p}<0.01)$, docetaxel $(r=0.92, p<0.01)$, topotecan $(r=0.84, p<0.01)$, gemcitabine $(r=0.86, p<0.01)$, and doxorubicin $(r=0.40, p<0.046)$ was found to be significant. The correlation of results of ATP and DISC tests for carboplatin $(r=0.87, p<0.01)$, docetaxel $(\mathrm{r}=0.86, \mathrm{p}<0.01)$, paclitaxel $(\mathrm{r}=0.84, \mathrm{p}<0.01)$, topotecan $(r=0.95, p<0.01)$, gemcitabine $(r=0.95, p<0.01)$, and doxorubi$\operatorname{cin}(r=0.70, p<0.01)$ was also significant. No difference in chemosensitivity profiles of MTT, ATP, and DISC tests for carboplatin $(p=0.68)$, docetaxel $(p=0.82)$, paclitaxel $(p=0.89)$, topotecan $(p=0.32)$, and doxorubicin $(p=0.34)$ was found. However, for gemcitabine, the difference was significant $(\mathrm{p}<0.05)$.

The in vitro chemosensitivity results of chemotherapeutic agents was also studied in terms of prognostic parameters, including stage, grade, and histologic type. When results of stage I patients were compared with those of stage II and III patients, no significant difference was found between agents at

Table 1 . The in vitro chemosensitivity test results $(n=26)$

\begin{tabular}{|c|c|c|c|c|}
\hline Drug & Sensitive & $\begin{array}{c}\text { Moderately } \\
\text { sensitive }\end{array}$ & Resistant & $\begin{array}{c}\text { Extremely } \\
\text { resistant }\end{array}$ \\
\hline \multicolumn{5}{|l|}{ MTT } \\
\hline Carboplatin & $15(57.7 \%)$ & $3(11.5 \%)$ & $5(19.3 \%)$ & $3(11.5 \%)$ \\
\hline Docetaxel & $11(42.3 \%)$ & $5(19.3 \%)$ & $9(34.6 \%)$ & $1(3.8 \%)$ \\
\hline Paclitaxel & $12(46.1 \%)$ & $4(15.4 \%)$ & $8(30.8 \%)$ & $2(7.7 \%)$ \\
\hline Topotecan & $9(34.6 \%)$ & $6(23.1 \%)$ & $9(34.6 \%)$ & $2(7.7 \%)$ \\
\hline Gemcitabine & $8(30.8 \%)$ & $3(11.5 \%)$ & $11(42.3 \%)$ & $4(15.4 \%)$ \\
\hline Doxorubucine & $6(23.0 \%)$ & $2(7.7 \%)$ & $13(50.0 \%)$ & $5(19.3 \%)$ \\
\hline \multicolumn{5}{|l|}{ ATP-TCA } \\
\hline Carboplatin & $15(57.7 \%)$ & $3(11.5 \%)$ & $8(30.8 \%)$ & 0 \\
\hline Docetaxel & $11(42.3 \%)$ & $5(19.3 \%)$ & 7 (26.9\%) & $3(11.5 \%)$ \\
\hline Paclitaxel & $10(38.5 \%)$ & $8(30.8 \%)$ & $7(26.9 \%)$ & $1(3.8 \%)$ \\
\hline Topotecan & $10(38.5 \%)$ & $6(23.0 \%)$ & $10(38.5 \%)$ & 0 \\
\hline Gemcitabine & $4(15.4 \%)$ & $11(42.3 \%)$ & $10(38.5 \%)$ & $1(3.8 \%)$ \\
\hline Doxorubucine & $5(19.3 \%)$ & $8(30.8 \%)$ & $12(46.1 \%)$ & $1(3.8 \%)$ \\
\hline \multicolumn{5}{|l|}{ DISC } \\
\hline Carboplatin & $16(61.5 \%)$ & $4(15.4 \%)$ & $5(19.3 \%)$ & $1(3.8 \%)$ \\
\hline Docetaxel & $10(38.5 \%)$ & $6(23.0 \%)$ & $8(30.8 \%)$ & $2(7.7 \%)$ \\
\hline Paclitaxel & $15(57.7 \%)$ & $2(7.7 \%)$ & $7(26.9 \%)$ & $2(7.7 \%)$ \\
\hline Topotecan & $9(34.6 \%)$ & $6(23.1 \%)$ & $9(34.6 \%)$ & $2(7.7 \%)$ \\
\hline Gemcitabine & $4(15.4 \%)$ & $5(19.3 \%)$ & $12(46.0 \%)$ & $5(19.3 \%)$ \\
\hline Doxorubucine & $5(19.3 \%)$ & $4(15.4 \%)$ & $16(61.5 \%)$ & $1(3.8 \%)$ \\
\hline \multicolumn{5}{|c|}{$\begin{array}{l}\text { MTT: 3-(4,5-dimethylthiazol-2-yl)-2,5-diphenyltetrazolyum bromide; } \\
\text { ATP-TCA: adenosine triphosphate tumor chemosensitivity assay; DISC: } \\
\text { differential staining cytotoxicity }\end{array}$} \\
\hline
\end{tabular}



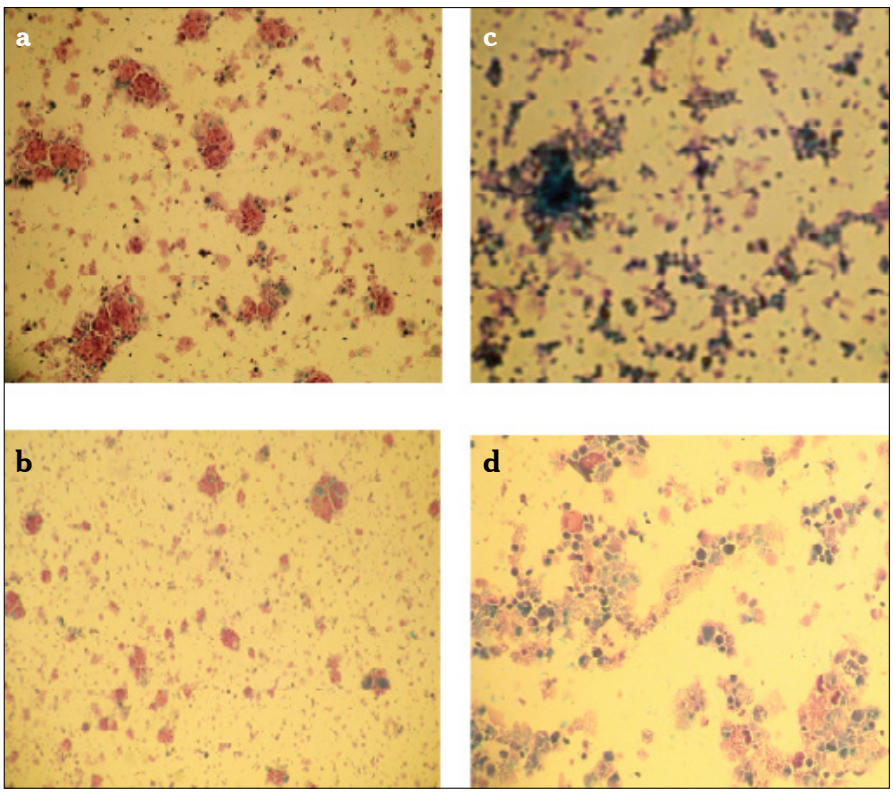

Figure 1. a-d. DISC assay results; control without drug (a), paclitaxel (sensitive) (b), control (extreme dose flourouracil) (c), Gemcitabine (resistant) (d)

early stage $(p=0.718)$; however, there was a significant difference for advanced stage (stage II and III) $(p=0.028)$. The difference between agents in the advanced stage was significant for doxorubicin and paclitaxel $(\mathrm{p}=0.048)$.

When grade 1 and 2 tumors together were compared with grade 3 tumor, no significant difference was found in grade 1 and $2(\mathrm{p}=0.221)$ and grade 3 groups with regard to chemotherapeutic agents $(\mathrm{p}=0.093)$.

In terms of histology, the series was categorized as serous and nonserous tumors. There were significant differences between agents for serous carcinoma $(p=0.004)$, but the difference was not significant in other histologic types $(p=0.573)$.

Since the standard first-line chemotherapy of ovarian carcinoma consists of paclitaxel and carboplatin, subgroup analysis was also performed for these drugs with respect to stage, grade, and histology. The in vitro efficacy of carboplatin and paclitaxel with the DISC assay according to the groups based on the stage of patients is presented in Figures 2 and 3. No statistically significant difference was found between stage Ic and stage II and III groups. When grade 1 and 2 tumors were grouped together and compared with grade 3 tumors, grade 1 and 2 patients were more sensitive to carboplatin and paclitaxel than grade 3 patients $(p=0.009)$. The efficacy of paclitaxel and carboplatin was studied in histologic type groups for serous and nonserous tumors; no statistical difference was found (Figures 4 and $5, \mathrm{p}>0.05)$.

\section{Discussion}

The main steps in the treatment of ovarian carcinoma are surgical resection of tumor mass, followed by employment of combination chemotherapy. The selection of chemotherapeutic agents for ovarian carcinoma is based on the results of previous

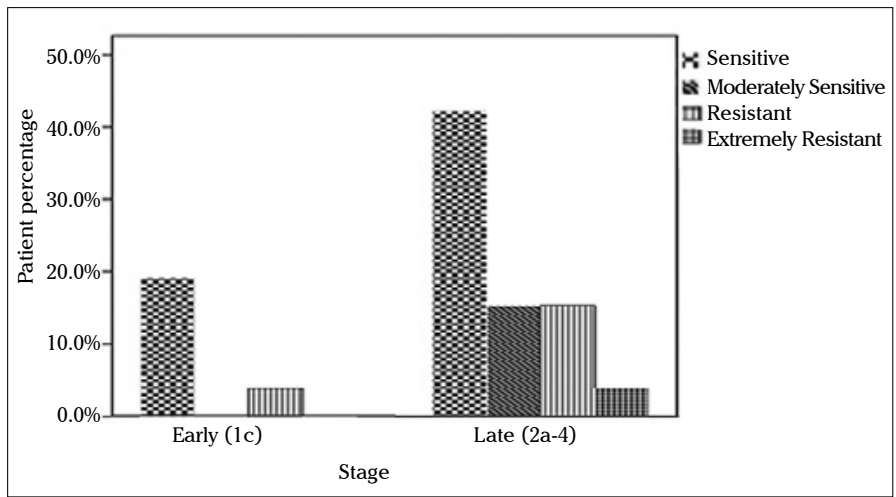

Figure 2. In vitro chemosensitivity of carboplatin according to DISC assay versus stage

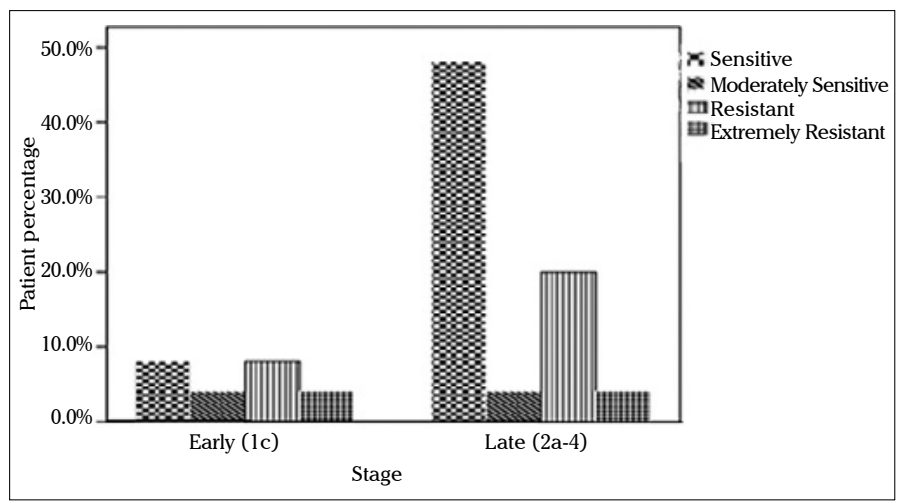

Figure 3. In vitro chemosensitivity of paclitaxel according to DISC assay versus stage

clinical trials. Despite advances in therapy, progression of the disease and even mortality are still problems originating from drug resistance. For an attempt to ameliorate the response to current treatment modalities, in vitro chemosensitivity assays have been developed for predetermining the possible effectivity (or ineffectivity) of chemotherapeutic agents prior to their administration to a patient. Several methods have been invented to foresee the effectivity of the treatment; however, debate still continues for the optimal application of these technologies. Sensitivity testing in primary ovarian cancer to individualize treatment remains an active area of interest. Furthermore, in recurrent ovarian cancer, there is lack of large prospective studies in which patients are randomized between standard therapy and assay directed therapy to show whether the directed therapy improves OS. However, some prospective and retrospective studies are promising that assay-directed therapy may offer a survival benefit $(17,18)$. In addition to the selection of effective drug for therapy, in vitro chemosensitivity assays are used for the prevention of unintended toxicity by the use of an inefficient chemotherapeutic agent.

Prognostic parameters, including stage, grade, and histologic type, were also studied with the scope of identifying subgroups in which in vitro chemosensitivity assays would be more useful. In addition, it particularly seems to be useful according to our study in advanced serous tumors. Furthermore, standard 


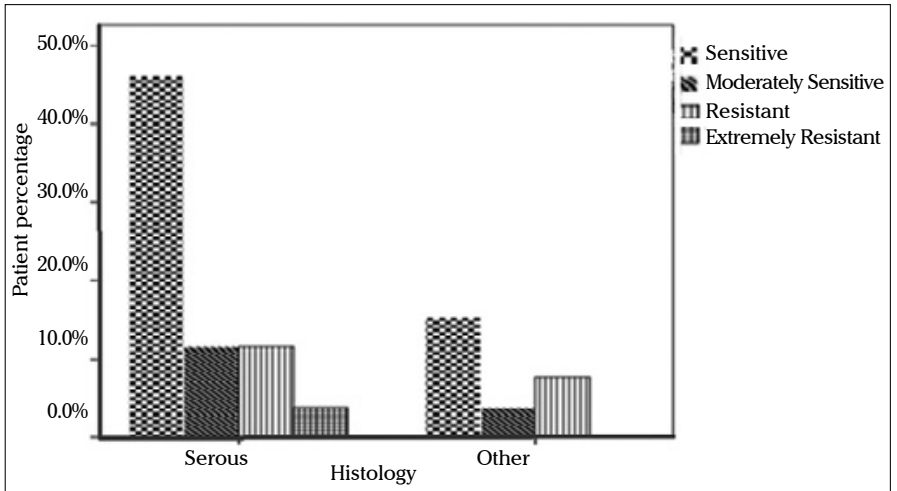

Figure 4. In vitro chemosensitivity of carboplatin according to DISC assay versus histologic type

chemotherapy seems to be more effective in low-grade tumors, as an expected result.

The present study is based on three different assays (MTT, ATP, and DISC assays) for the determination of in vitro chemosensitivity. ATP and MTT assays have been used to predict chemosensitivity in the literature with good clinical correlation (19, 20). However, they are unable to discriminate the tumor cells with other cells in the specimen and have difficulty in the determination of the level of cytotoxicity. The ratio of dead cells to all cells in the DISC assay gives information about the sensitivity and can distinguish contaminating cells, thereby avoiding false results. In addition, metabolically inactive cells can be seen as alive in the DISC assay, whereas in other tests with metabolic end-points, they may be misleadingly counted as dead. However, the DISC assay is a time-consuming and somewhat subjective method.

The correlation of the quantitative values of the ATP and MTT assay spectrophotometrically with those of the DISC assay for chemotherapeutic assay was studied in a series of 26 primary ovarian cancer cases. It has been found that results of these three different tests were not different from each other for the drugs paclitaxel, carboplatin, docetaxel, topotecan, and doxorubicin. However, gemcitabine represents an exception to this finding, and no explanation could be found for that difference. One of the important findings of this study is that in vitro chemosensitivity testing can be performed successfully with one of these three tests because the results were found to be similar.

The clinical utility of in vitro chemosensitivity assays has been evaluated in clinical trials that studied the relationship between in vitro test results and the patient's actual clinical response to that chemotherapeutic agent. For ovarian cancer, the negative predictive value ranged from $62 \%$ to $100 \%$, whereas the positive predictive value was between $58 \%$ and $91 \%$. Negative predictive values are generally higher than positive predictive values, which suggests that in vitro assays are better in identifying ineffective drugs (20).

In conclusion, in vitro chemosensitivity can be determined in ovarian carcinoma with one of ATP, MMT, and DISC assays before the initiation of chemotherapy. In vitro assays seem to be more useful in subgroups with advanced stage and serous histology. Larger prospective randomized studies are required to support the routine clinical use of in vitro chemosensitivity assays.

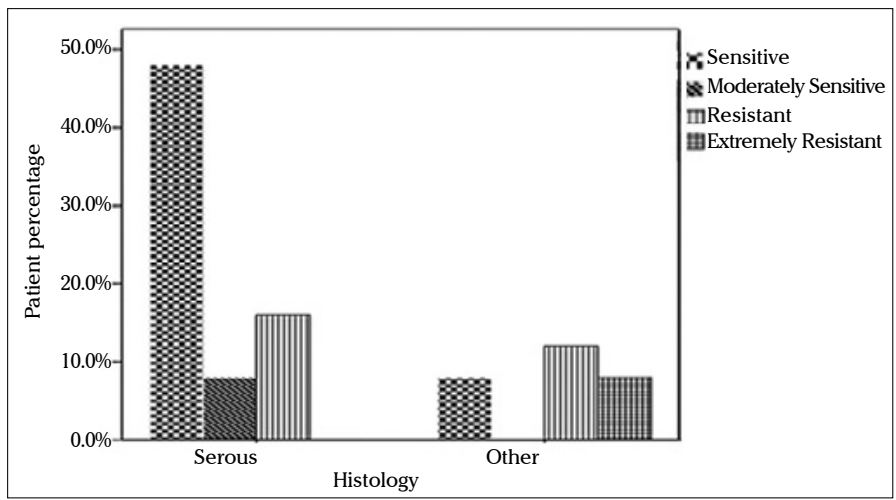

Figure 5. In vitro chemosensitivity of paclitaxel according to DISC assay versus histologic type

Ethics Committee Approval: Ethics committee approval was received for this study from the ethics committee of Hacettepe University.

Informed Consent: Written informed consent was obtained from patients who participated in this study.

Peer-review: Externally peer-reviewed

Author Contributions: Concept - Z.S.T., A.K.D., B.T.; Design - Z.S.T., A.K.D.; Supervision - Z.S.T.; Funding - Z.S.T., A.K.D.; Materials - A.K.D., B.T., A.U.; Data Collection and/or Processing - B.T., A.U., A.K.D.; Analysis and/or Interpretation - B.T.; Literature Review - B.T., Z.S.T.; Writer - B.T., G.B., I.S.; Critical Review - B.T., Z.S.T., G.B., I.S.

Conflict of Interest: No conflict of interest was declared by the authors.

Financial Disclosure: The study is supported by The Scientific and Technological Research Council of Turkey (TUBITAK Project-110S101).

\section{References}

1. Jemal A, Bray F, Center MM, Ferlay J, Ward E, Forman D. Global cancer statistics. CA Cancer J Clin 2011; 61: 69-90. [CrossRef]

2. Quirk JT, Natarajan N. Ovarian cancer incidence in the United States, 1992-1999. Gynecol Oncol 2005; 97: 519-23. [CrossRef]

3. Young RC, Walton LA, Ellenberg SS, Homesley HD, Wilbanks GD, Decker DG, et al. Adjuvant therapy in stage I and stage II epithelial ovarian cancer. Results of two prospective randomized trials. N Engl J Med 1990; 322: 1021-7. [CrossRef]

4. Stewart L. Chemotherapy for advanced ovarian cancer. Advanced Ovarian Cancer Trialists Group. Cochrane Database Syst Rev 2000; 2: CD001418.

5. Sarosy GA, Hussain MM, Seiden MV, Fuller AF, Nikrui N, Goodman A, et al. Ten-year follow-up of a phase 2 study of dose-intense paclitaxel with cisplatin and cyclophosphamide as initial therapy for poor-prognosis, advanced-stage epithelial ovarian cancer. Cancer 2010; 116: 1476-84. [CrossRef]

6. Copeland LJ, Bookman M, Trimble E. Clinical trials of newer regimens for treating ovarian cancer: the rationale for Gynecologic Oncology Group Protocol GOG 182-ICON5. Gynecol Oncol 2003; 90: S1-7. [CrossRef]

7. Coleman RL, Monk BJ, Sood AK, Herzog TJ. Latest research and treatment of advanced-stage epithelial ovarian cancer. Nat Rev Clin Oncol 2013; 10: 211-24. [CrossRef]

8. Sevin BU, Perras JP. Tumor heterogeneity and in vitro chemosensitivity testing in ovarian cancer. Am J Obstet Gynecol 1997; 176: 759-66; discussion 766-8. [CrossRef] 
9. Black MM, Speer FD. Further observations on the effects of cancer chemotherapeutic agents on the in vitro dehydrogenase activity of cancer tissue. J Natl Cancer Inst 1954; 14: 1147-58.

10. Kern DH. Tumor chemosensitivity and chemoresistance assays. Cancer 1997; 79: 1447-50. [CrossRef]

11. Cree IA. Chemosensitivity and chemoresistance testing in ovarian cancer. Curr Opin Obstet Gynecol 2009; 21: 39-43. [CrossRef]

12. Tuefferd M, Couturier J, Penault-Llorca F, Vincent-Salomon A, Broet P, Guastalla JP, et al. HER2 status in ovarian carcinomas: a multicenter GINECO study of 320 patients. PloS one 2007; 2: e1138. [CrossRef]

13. Tewari K, Manetta A. In vitro chemosensitivity testing and mechanisms of drug resistance. Curr Oncol Rep 1999; 1: 77-84. [CrossRef]

14. Rutherford T, Orr J Jr., Grendys E Jr., Edwards R, Krivak TC, Holloway $\mathrm{R}$, et al. A prospective study evaluating the clinical relevance of a chemoresponse assay for treatment of patients with persistent or recurrent ovarian cancer. Gynecol Oncol 2013; 131: 362-7. [CrossRef]

15. Tian C, Sargent DJ, Krivak TC, Powell MA, Gabrin MJ, Brower SL, et al. Evaluation of a chemoresponse assay as a predictive marker in the treatment of recurrent ovarian cancer: further analysis of a prospective study. Br J Cancer 2014; 111: 843-50. [CrossRef]
16. Previs R, Leath CA 3rd, Coleman RL, Herzog TJ, Krivak TC, Brower $\mathrm{SL}$, et al. Evaluation of in vitro chemoresponse profiles in women with Type I and Type II epithelial ovarian cancers: An observational study ancillary analysis. Gynecol Oncol 2015; 138: 267-71. [CrossRef]

17. Cree IA, Kurbacher CM, Lamont A, Hindley AC, Love S. A prospective randomized controlled trial of tumour chemosensitivity assay directed chemotherapy versus physician's choice in patients with recurrent platinum-resistant ovarian cancer. Anticancer Drugs 2007; 18: 1093-101. [CrossRef]

18. Loizzi V, Chan JK, Osann K, Cappuccini F, DiSaia PJ, Berman ML. Survival outcomes in patients with recurrent ovarian cancer who were treated with chemoresistance assay-guided chemotherapy. Am J Obstet Gynecol 2003; 189: 1301-7. [CrossRef]

19. Blumenthal RD. Chemosensitivity. In: Blumenthal RD, editor. Methods in Molecular Medicine. 1. New Jersey: Humana Press; 2005. [CrossRef]

20. Fruehauf JP. In vitro assay-assisted treatment selection for women with breast or ovarian cancer. Endocr Relat Cancer 2002; 9: 171-82. [CrossRef] 